\title{
La regulación fotovoltaica y solar termoeléctrica en España*
}

\author{
Pere Mir \\ Universitat de Lleida
}

\section{Resumen}

Tras unos años de escaso éxito en la promoción de la generación solar en España, la potencia fotovoltaica vivió un boom en los años 2007 y 2008. La necesidad de controlar el costo económico resultante, dio lugar a una especial densidad normativa en 2009 y 2010. En concreto, se tomaron dos tipos de medidas: para la nueva capacidad, estrictos cupos e importantes rebajas de las tarifas y, para la ya autorizada, una destacada reducción del número de horas con derecho a prima. Algunas de estas medidas fueron consideradas retroactivas por el sector, de forma que han sido recurridas ante los tribunales. Recién iniciado el año 2012, un nuevo Real decreto-ley estableció una moratoria sine die de la generación solar, el futuro de la cual queda por consiguiente en entredicho.

Palabras clave: regulación de fuentes renovables de electricidad, generación fotovoltaica, generación solar termoeléctrica.

Clasificación JEL: Q41, Q42, Q51.

\begin{abstract}
For years the promotion of solar power in Spain had no success. But in 2007 and 2008 the PV generation experienced a boom. The control of the ensuing economic burden, led to an intensive regulatory activity in 2009 and 2010. Two measures were specifically taken: for new capacity, strict quotas and significant cuts in tariffs, and, with respect the operating capacity, an important reduction in the number of eligible hours. Some of these measures were considered retroactive by the industry, so that have been contested in the courts. Just started 2012, a new Royal Decree established a solar generation moratorium for an indefinite period. As a result, the future of solar generation in Spain is compromised.
\end{abstract}

Keywords: renewables regulation policies, photovoltaics, concentrating solar power.

JEL classification: Q41, Q42, Q51.

\section{Introducción}

Este artículo recorre las principales vicisitudes de la generación fotovoltaica (apartados 2 a 4) y de la solar termoeléctrica (punto 5) desde finales del siglo pasado hasta 2011. Sigue el apartado 6 consagrado a las decisiones regulatorias tomadas en 2012 que da paso a unas breves conclusiones finales.

* Este artículo es un breve resumen del capítulo 6 del libro Economía de la generación solar eléctrica. La regulación fotovoltaica y solar termoeléctrica en España, de próxima publicación por Editorial Cívitas. Se remite al lector a esta fuente para recabar mucha más información al respecto. 
Con la aprobación de la Ley del Sector Eléctrico (L 54/1997) se sentaron las bases de la política de apoyo a la generación solar, entre muchos otros aspectos. Un año después, el RD 2818/1998 establecía el pago de una tarifa o prima (a elegir) por kWh y, además, animaba a las instalaciones fotovoltaicas, aun cuando tuvieran titulares distintos, a compartir puntos de conexión a la red. Se generalizó, pues, la yuxtaposición de plantas de pequeña potencia denominadas huertos solares. Ello, por una parte, garantizaba obtener la máxima retribución y, por la otra, permitía aprovechar economías de escala compartiendo equipos y líneas de evacuación (CNE, 2008: p. 25, y De la Hoz, 2010: pp. 2559-2561).

Con el RD 436/2004, las tarifas pasaron a ser establecidas como un porcentaje de la tarifa eléctrica media o de referencia pagada por los abonados. También se mantuvo la posibilidad de los huertos solares, aunque su tamaño ascendió hasta los $100 \mathrm{~kW}_{\mathrm{p}}{ }^{1}$.

\section{El RD 661/2007}

El RD 661/2007 eliminó cualquier atisbo de incertidumbre con respecto a la retribución de las plantas fotovoltaicas: la tarifa inicial era fija y su valor, para las plantas ya autorizadas, se actualizaría todos los años según la inflación, menos una pequeña deducción porcentual. La norma disponía, además, un descenso parcial de la tarifa tras veinticinco años. La combinación de una tarifa perfectamente conocida y un método de actualización inequívoco y fácil de aplicar, junto con la garantía temporal de largo plazo, condujo la inversión en plantas fotovoltaicas a un grado de seguridad poco común.

El RD establecía que el marco retributivo sería revisado en 2010 y, de ahí en adelante, cada cuatro años. La norma, sin embargo, también preveía otro criterio de revisión: alcanzar un determinado volumen de potencia instalada. En el caso fotovoltaico, el objetivo de referencia eran $371 \mathrm{MW}$. Alcanzado el 85 por 100 de este objetivo, se ponía en marcha el proceso de revisión de las tarifas (art. 22.1). Este empezaba con el anuncio del plazo máximo de prórroga del esquema retributivo vigente, cuya duración no podía ser inferior a un año. Una vez vencido este periodo extra, las nuevas plantas pasarían a percibir una remuneración equivalente al precio final horario del mercado mayorista de la electricidad (si habían optado por la tarifa, como era habitual en el sector fotovoltaico). En otras palabras, la retribución caía en picado dado que los precios de mercado eran muy inferiores a la tarifa.

En los doce meses anteriores a la entrada en vigor del RD 661/2007, la capacidad instalada había ido creciendo a un ritmo medio mensual de $17 \mathrm{MW}$. Puesto que, en mayo de 2007, había 261 MW fotovoltaicos conectados a la red, el objetivo del RD se alcanzó en apenas unas semanas: al mes siguiente, la CNE ya contabilizó

1 Para encuadrar estas medidas dentro de la política de regulación de las fuentes renovables de electricidad en España, véanse DEL RÍO y GUAL (2007) y DEL RÍO (2008). 
326 MW. Queda claro, por lo tanto, el intento del legislador de controlar la expansión de la generación fotovoltaica, probablemente preocupado por su elevado coste. Sin embargo, la prórroga fijada (que fue anunciada en septiembre de 2007) era muy larga y estaba abocada, si no entraba en vigor un nuevo esquema tarifario, al fin del régimen retributivo preferencial.

Para seguir con detalle lo acaecido en el plazo transitorio, véase el Gráfico 1, que muestra la capacidad fotovoltaica instalada mes a mes desde febrero de 2006 hasta terminar $2011^{2}$.

\section{GRÁFICO 1 \\ POTENCIA FOTOVOLTAICA MENSUAL INSTALADA (FEBRERO 2006-2011)}

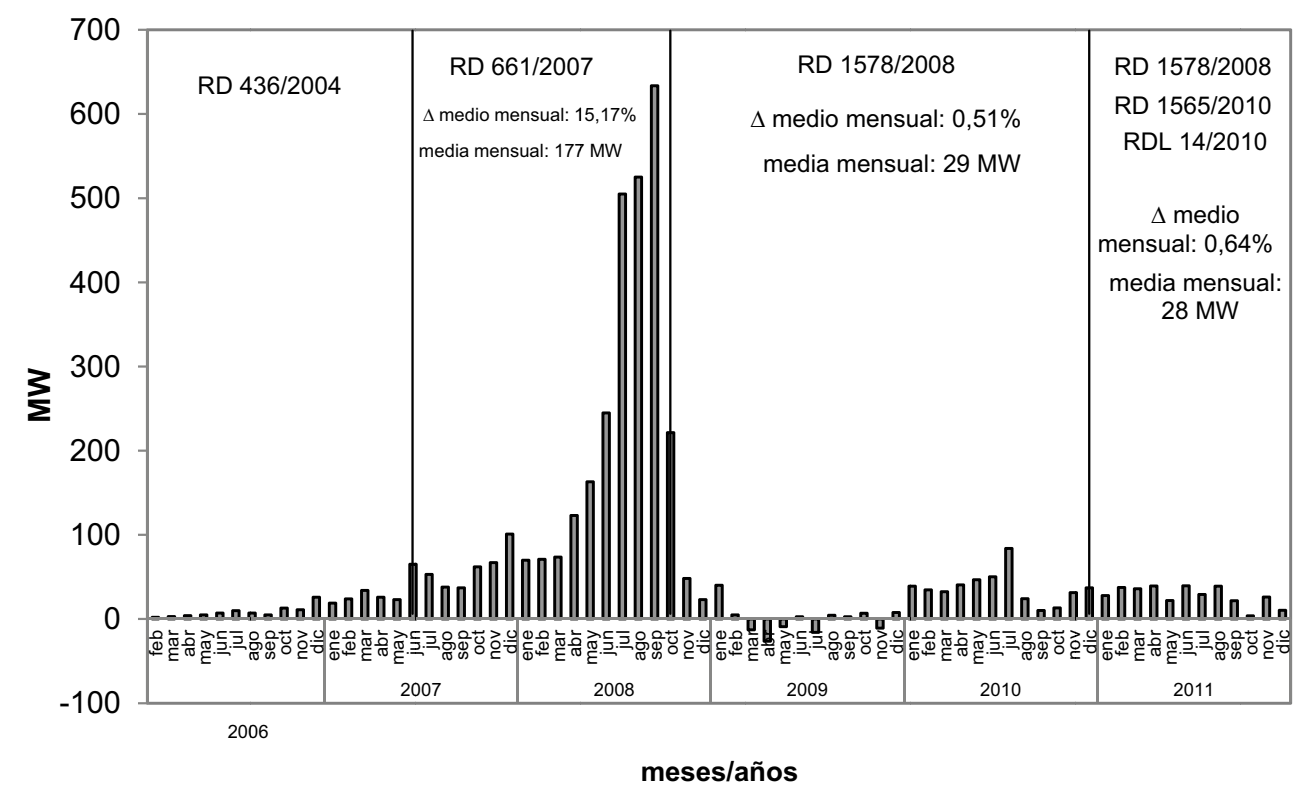

Como se observa, durante el otoño de 2007 y posterior invierno, la potencia empezó a crecer, aunque de forma un tanto irregular. Sin embargo, habida cuenta del lapso necesario para la redacción y tramitación de los proyectos, estaba claro que la expansión era cada vez más rápida. El punto álgido fueron los meses de junio y

${ }^{2}$ Elaboración propia en base a los datos publicados por la CNE con fecha de actualización del 17/2/2012. Según explica esta fuente, por potencia instalada se entiende la potencia bruta conectada a la red instalada esté, o no, facturando energía. Hay que advertir que los datos publicados en 2012 reducen de forma significativa cifras anteriores: por ejemplo, la potencia instalada en 2010 es entre 300 y 500 MW menor, magnitud que se duplica si la comparación se extiende hasta octubre de 2011. De hecho, desde mediados de 2010, la potencia fotovoltaica consignada es entre un 10 y un 16 por 100 menor. A tenor de las explicaciones dadas por la CNE, la modificación se debería a la puesta al día de las facturaciones, al efecto de las inspecciones llevadas a cabo y, sobretodo, al haber considerado por separado los datos de la generación solar termoeléctrica. Los informes mensuales de ventas de energía del régimen especial están disponibles en www.cne.es. 
septiembre de 2008 cuando se llegaron a instalar más de 500 MW mensuales. A finales de septiembre, la capacidad inscrita era de $3.116 \mathrm{MW}$. El boom que estaba viviendo el sector fotovoltaico español carecía de precedentes y dejaba boquiabierto al mundo entero.

Conocida la evolución de la potencia, el Gráfico 2 muestra la evolución del volumen de prima equivalente percibida por el sector fotovoltaico, tanto en términos absolutos como por kWh. A simple vista queda claro el enorme impacto económico del boom fotovoltaico.

\section{GRÁFICO 2 \\ EVOLUCIÓN DE LA PRIMA FOTOVOLTAICA EQUIVALENTE}

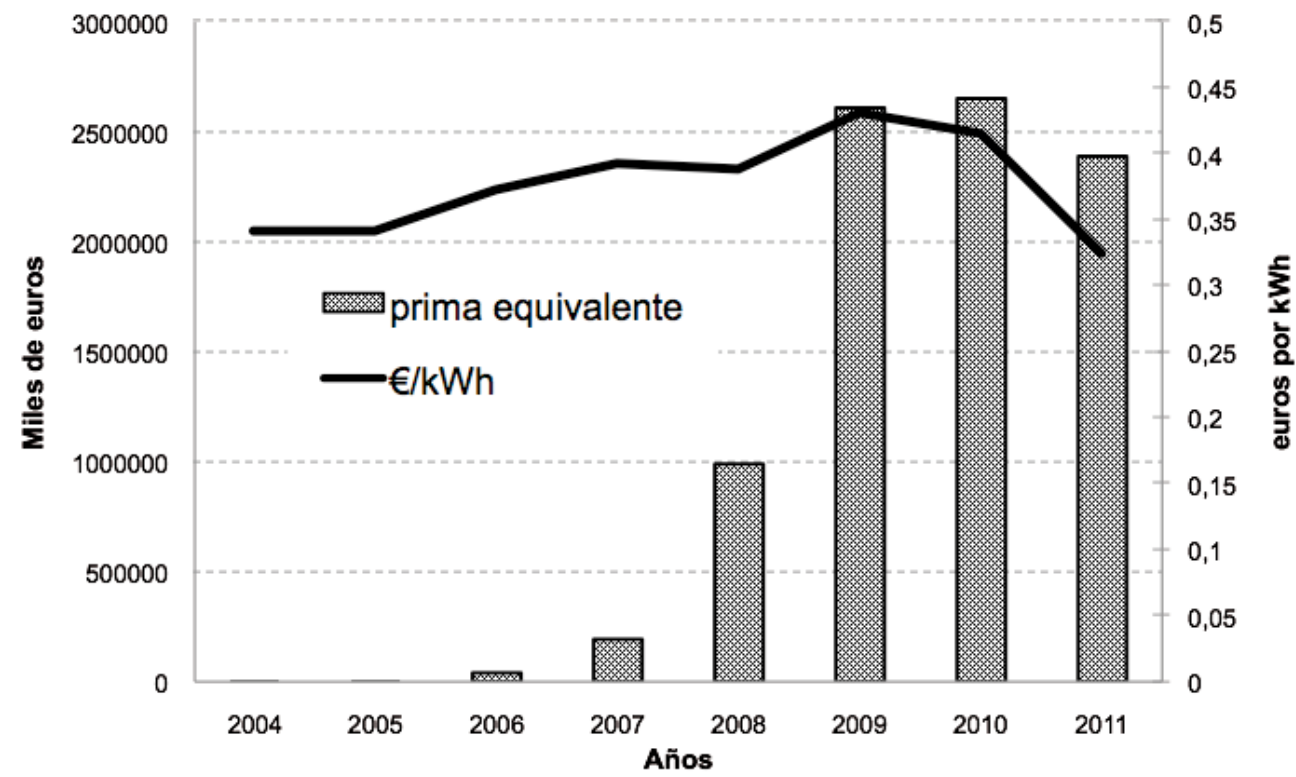

El Cuadro 1 (elaboración propia de datos de la CNE) muestra cómo el alto coste del $\mathrm{kWh}$ fotovoltaico provoca que, el peso de la prima equivalente asociada, sea cuatro o más veces más intenso que su contribución al mix de generación de las técnicas del régimen especial. 


\section{CUADRO 1}

\section{EL PESO DE LA TARIFAS Y LA CONTRIBUCIÓN DE LA GENERACIÓN FOTOVOLTAICA}

\begin{tabular}{|c|c|c|}
\hline & $\begin{array}{c}\text { Prima fotovoltaica/prima } \\
\text { equivalente (\%) }\end{array}$ & $\begin{array}{c}\text { Aporte fotovoltaico al mix régimen } \\
\text { especial (\%) }\end{array}$ \\
\hline 2004 & 0,494 & 0,038 \\
\hline 2005 & 1,123 & 0,079 \\
\hline 2006 & 2,235 & 0,205 \\
\hline 2007 & 8,502 & 0,849 \\
\hline 2008 & 29,346 & 3,698 \\
\hline 2009 & 42,844 & 7,621 \\
\hline 2010 & 37,205 & 7,009 \\
\hline 2011 & 34,816 & 8,009 \\
\hline
\end{tabular}

Vistos los hechos, falta por discernir los factores que los motivaron. Para empezar, un error habitual es atribuir la responsabilidad principal, o incluso exclusiva, del boom a la excesiva tarifa fotovoltaica. Sin embargo, se trata de un diagnóstico precipitado, superficial. El Gráfico 3 exhibe la trayectoria temporal trimestral de las tarifas desde finales de 1998 hasta finales de 2011. Se han indicado, también, los periodos de vigencia de los diversos reales decretos que inciden en la técnica fotovoltaica (elaboración propia a partir de las bases de datos de la CNE).

Si se pone atención en el periodo abarcado por los reales decretos 436/2004 y 661/2007, se observa que la retribución para las instalaciones fotovoltaicas más comunes se mantuvo justo por debajo de los $45 \mathrm{cts} \square / \mathrm{kWh}$ desde inicios de 2006 . Por consiguiente, responsabilizar sólo a las tarifas implica pasar por alto el hecho de que el boom tardó casi dos años en arrancar (o más, si se tiene en cuenta que las tarifas del RD 436/2004 apenas eran inferiores). Habrá de confeccionarse, pues, un cuadro causal más complejo.

Tras evaluar la situación económica del momento y los rasgos técnicos de la generación fotovoltaica, diversas serían los factores que animaron el boom. A saber, 


\section{GRÁFICO 3}

EVOLUCIÓN DE LAS TARIFAS FOTOVOLTAICAS (1998-2011)

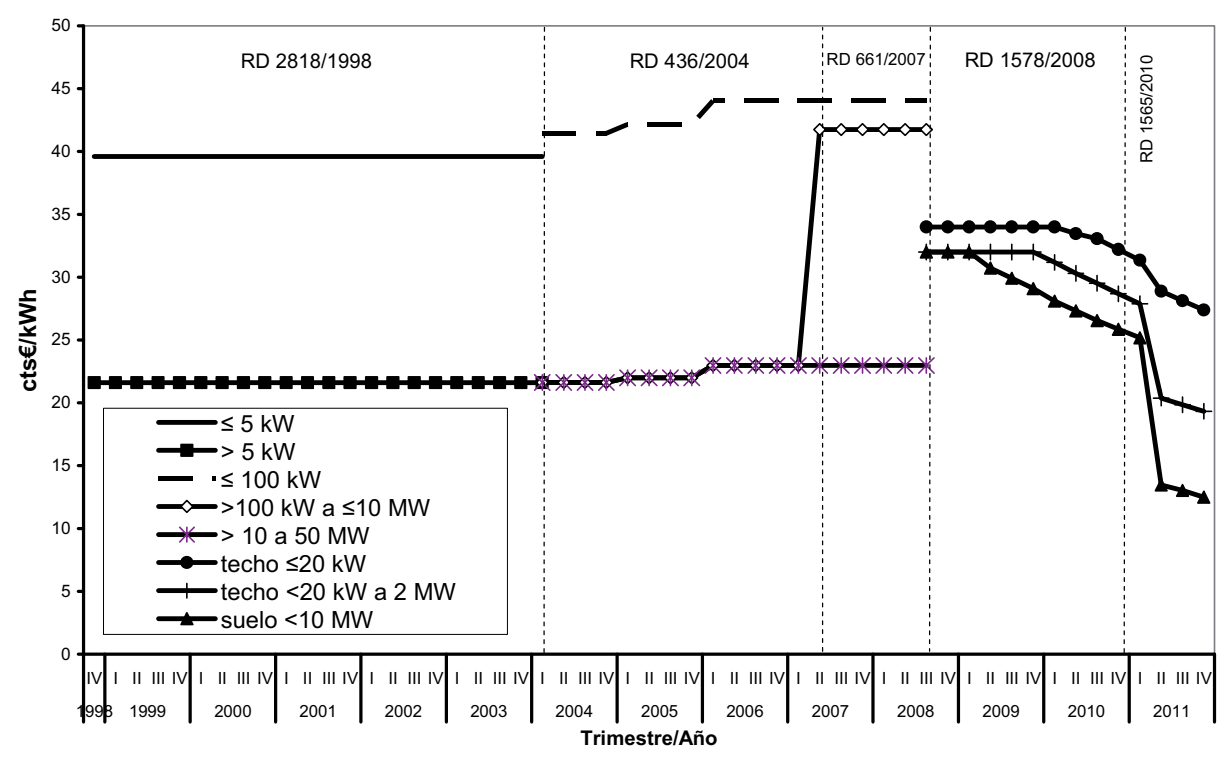

1. Los efectos de la progresiva debilidad del sistema financiero internacional, se vieron agravados en España por el empeoramiento de las expectativas del sector de la construcción. Ante la crisis que se avecinaba, había que diversificar los excedentes acumulados en los años anteriores. La inversión en energías renovables, rentable y muy segura, atrajo a todo tipo de inversionistas: desde grandes corporaciones a profesionales y pequeños empresarios, pasando por fondos de inversión y de pensiones, nacionales y extranjeros.

2. Las instituciones financieras publicitaron la inversión en plantas fotovoltaicas como un magnífico producto financiero. La gran capacidad de comunicación de bancos y cajas explica que tales virtudes, nada desdeñables ante el empeoramiento de las expectativas, se difundieran con rapidez. La inversión total en las más de 50 mil plantas fotovoltaicas existentes, se estima en unos 20 mil millones de euros, un 75 por 100 de los cuales aportados por la banca española o extranjera.

3. A menudo, las instituciones financieras otorgaron facilidades crediticias a particulares a través del método del project finance. Se constituía, pues, una pequeñísima sociedad promotora del huerto solar que, a pesar de operar con un alto nivel de endeudamiento con respecto a sus recursos propios, la garantía tarifaria aseguraba la obtención de un flujo neto de caja para afrontar el servicio del préstamo. La duración de los créditos se extendía por una década o poco más. 
4. El carácter modular de las instalaciones fotovoltaicas facilitó que la inversión fuese asequible a infinidad de pequeños inversores.

5. Agrupar conjuntos de módulos de poca potencia, cada uno con su denominación social y su/s titular/es, resultaba más fácil que constituir grandes parques cuyo capital social estuviese repartido entre múltiples accionistas. De esta forma, el sistema de los huertos solares facilitó el crecimiento del sector.

6. La perspectiva de una regulación más severa con posterioridad al mes de septiembre de 2008, animó la carrera de los meses anteriores. No solamente el largo plazo transitorio terminaba de forma abrupta, sino que, en fecha tan temprana como septiembre de 2007, se divulgó un borrador de nuevo real decreto que incluía una clara rebaja de tarifas.

7. Otra razón a tener en cuenta es que pueden ubicarse placas fotovoltaicas por doquier. España cuenta con miles de $\mathrm{km}^{2}$ con elevada radiación solar. Gran parte de este territorio está poco poblado y consiste en terrenos de escaso o modesto valor agrícola. La única restricción importante es el punto de conexión. Aun así, la disponibilidad de emplazamientos es ingente.

8. Algunos gobiernos regionales ampliaron las ventajas económicas a los promotores fotovoltaicos, otros simplificaron las condiciones para su aprobación y, en algunos casos, a medida que se acercaba la fecha límite, se actuó con poco rigor administrativo.

9. Existen razones sólidas para creer que se obtenía una rentabilidad superior a la prevista por el regulador. Mientras la memoria económica del RD $661 / 2007$ preveía una TIR situada entre un suelo del 5 por 100 y un techo del 9 por 100, las empresas instaladoras que peinaban el territorio en busca de los mejores emplazamientos, entusiasmaban a los posibles inversores con rentabilidades de entre el 10 y el 15 por 100 . Las razones de ello serían las dos siguientes:

- Dado que la potencia de un parque se define por la capacidad nominal del inversor, una práctica común fue dimensionar el campo de módulos un 1520 por 100 por encima de ésta. Con ello aumentaba la cantidad de energía vertida a la red y, de ahí, la rentabilidad de la inversión.

- La continua mejora de las placas ha permitido generar más energía para una misma potencia nominal lo cual, junto con el descenso de sus precios desde 2009, habría llevado a la sustitución de módulos, con apenas unos pocos años de antigüedad, por otros de mayor potencia y más baratos. Dado el vacío regulatorio al respecto, esta práctica no acarreaba una menor retribución, con lo que se alcanzaban mayores niveles de rentabilidad (ASIF, 2011, p. 29).

Ambas circunstancias aumentaron los niveles de producción, o su equivalente en número de horas de funcionamiento a potencia nominal (factor de carga), utilizados en el cálculo de la rentabilidad de los parques. 
10. Durante aquellos meses, la cotización del euro frente al dólar fue muy elevada. La coyuntura era, pues, propicia a las importaciones masivas de paneles y otros componentes, como muestra la tabla 2 (datos procedentes de Funk et al., 2010, p. 53). Se constata que el saldo en 2007 y 2008 fue muy negativo.

\section{CUADRO 2}

\section{COMERCIO EXTERIOR ESPAÑOL DE CÉLULAS Y MÓDULOS FOTOVOLTAICOS (millones de \$)}

\begin{tabular}{|l|c|c|r|r|r|r|r|}
\hline & 1996 & 2000 & 2004 & 2005 & \multicolumn{1}{c|}{2006} & \multicolumn{1}{c|}{2007} & \multicolumn{1}{c|}{2008} \\
\hline Exportaciones & 14,5 & 54,3 & 283,1 & 266,7 & 258,2 & 190,0 & 363,6 \\
\hline Importaciones & 19,7 & 31,3 & 62,8 & 198,5 & 942,1 & $3.126,3$ & $7.946,5$ \\
\hline Saldo & $-5,2$ & 23 & 220,3 & 68,2 & $-683,9$ & $-2.036,3$ & $-7.582,9$ \\
\hline
\end{tabular}

Este flujo de importaciones coincidió con la insuficiencia temporal de polisilicio (material base para las células) debido a la fuerte demanda internacional, lo que presionó al alza los precios de los módulos (hasta principios de 2009). A su vez, se sospecha que los fabricantes cargaban precios más altos a los equipos destinados al boyante mercado español. Una práctica que, probablemente, también siguieron distribuidores y promotores domésticos. El alto precio que se pagó por los módulos, recortó la rentabilidad de los parques aunque no lo suficiente como para desistir de los proyectos.

La combinación de todos estos factores económicos y técnicos, explica la extraordinaria evolución del sector fotovoltaico durante buena parte del año 2008. A finales de septiembre, la tónica cambió drásticamente.

\section{EI RD 1578/2008}

El RD 1578/2008 fue el primero en clasificar las instalaciones fotovoltaicas según ubicación, dando una ligera ventaja retributiva a las de techo. Además, con el fin de controlar el crecimiento del sector, se estableció un sistema de convocatorias trimestrales, cuyo grado de éxito en satisfacer el cupo de nueva capacidad fotovoltaica admitida, determinaba el precio del $\mathrm{kWh}$ que percibirían los proyectos inscritos. Para ajustar las solicitudes de nuevas instalaciones y el cupo de potencia de cada convocatoria, se creó el Registro de preasignación de retribución (art. 4). El mecanismo diseñado suponía un descenso interanual de las tarifas del 10 por 100. Para las plantas en activo, la tarifa inicial percibida se actualizaba durante 25 años. Hay que añadir que el artículo 10.2 ponía punto final a los huertos solares.

La entrada en vigor del 1578/2008, con su estricto cupo y descenso asociado de las tarifas, junto con los efectos de la crisis económica, frenaron en seco la expansión 
de la capacidad fotovoltaica. De acuerdo con el Gráfico 4 (datos procedentes de la $\mathrm{CNE}$ ), no sólo la tendencia cambió de forma radical sino que, durante el primer año de vigencia de esta norma, el sector quedó casi paralizado.

Dada la estrechez de los cupos, a lo largo de las primeras convocatorias, cuyo calendario se retrasó, fue muy alto el número de proyectos de plantas de suelo que estuvieron a la espera. A su vez, los proyectos de techo no consiguieron cubrir su correspondiente cupo hasta finales de 2009 e inicios de 2010 por la falta de experiencia previa ${ }^{3}$.

\section{GRÁFICO 4 \\ EVOLUCIÓN DE LA POTENCIA FOTOVOLTAICA ACUMULADA (2006-2011)}

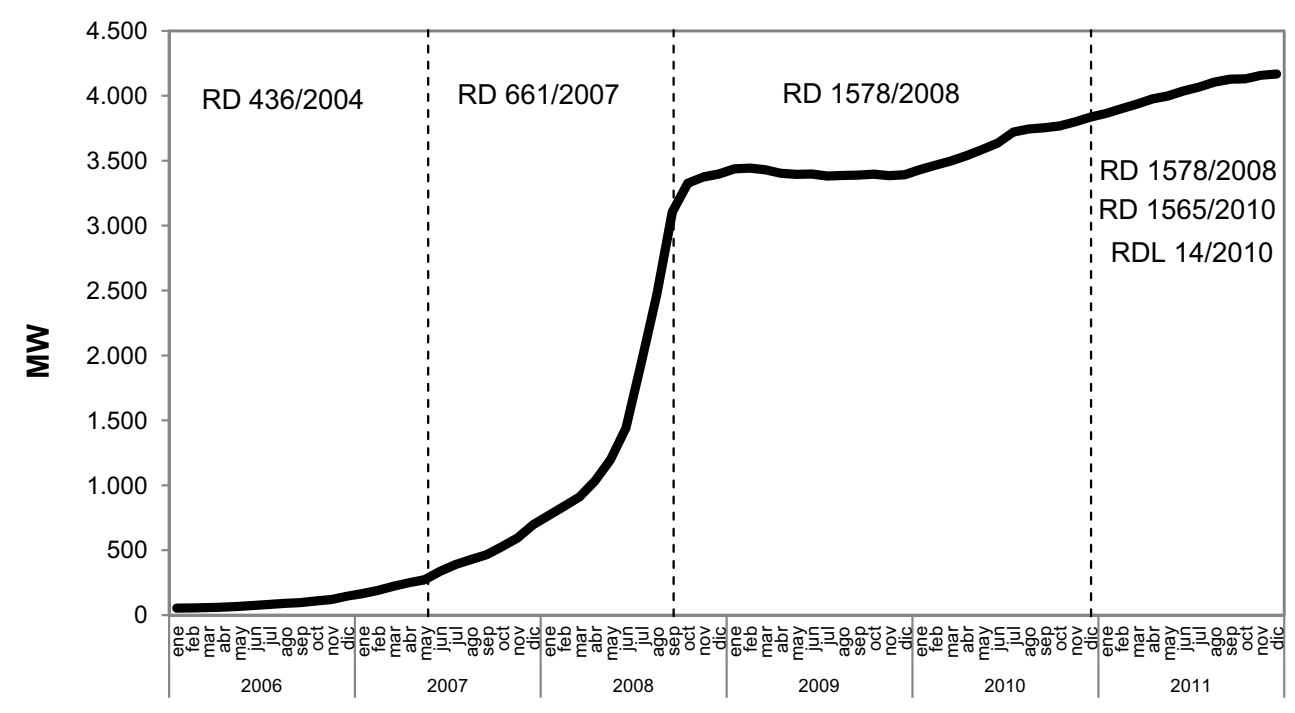

meses/años

El freno regulatorio y la crisis económica supusieron la pérdida de muchos puestos de trabajo. Si en el año 2006 la ocupación directa en el sector fotovoltaico se cifraba en alrededor de 6.300 personas (Jäger-Waldau, 2007, pp. 82-83), con el boom ascendió rápidamente para caer luego de la misma forma (véase Cuadro 3) (ASIF, 2011, p. 87).

Otro fenómeno del momento fueron los rumores acerca de prácticas especulativas con los derechos de instalación. Para evitarlas, los RD 661/2007 y RD 1.578/2008 exigieron avales. También ha habido fondos de inversión y de pensiones, nacionales

\footnotetext{
${ }^{3}$ Las condiciones económicas que fijó el RD 1578/2008, no provocaron una severa reducción de la rentabilidad de las instalaciones, debido al descenso de los precios de los módulos (MIR, 2009, pp. 133-136).
} 
e internacionales, que han adquirido parques en funcionamiento según la retribución establecida en tales reales decretos. Parece que se han llegado a pagar entre $6.000 \mathrm{y}$ $8.200 \square / \mathrm{kW}$ (Cinco Días, 4/11/2008, p. 5).

\section{CUADRO 3}

PUESTOS DE TRABAJO EN EL SECTOR FOTOVOLTAICO ESPAÑOL

\begin{tabular}{|c|c|c|c|}
\hline \multirow{2}{*}{} & \multicolumn{3}{|c|}{ Puestos de trabajo } \\
\cline { 2 - 4 } & Fijos & Temporales & Total \\
\hline 2008 & 15.400 & 26.300 & 41.700 \\
\hline 2009 & 11.300 & 2.600 & 13.900 \\
\hline 2010 & 11.600 & 2.100 & 13.700 \\
\hline 2011 & 10.600 & 1.600 & 12.100 \\
\hline
\end{tabular}

Otra vicisitud más fue el fraude con la fecha de inicio de actividad de las plantas fotovoltaicas. Algunos promotores, con la colaboración de instancias administrativas, llegaron a falsificar dicha fecha para que su instalación quedase acogida a las mejores condiciones económicas del RD 661/2007. A finales de 2008, la CNE inició una campaña de inspección que, en julio de 2009, se extendió a las plantas sujetas al RD 1578/2008, donde también se sospechaban irregularidades administrativas. Para reforzar el apoyo legal a esta campaña, el Ministerio de Industria hizo público un borrador de real decreto en enero de 2009, aunque hasta el 5 agosto del año siguiente no se promulgó el RD 1.003/2010. Esta norma creaba en el seno del Registro estatal del régimen especial una sub-sección singular: la de las instalaciones sin retribución primada. No obstante, las plantas irregularmente acogidas al RD 661/2007 que voluntariamente renunciasen a este régimen económico, ni tendrían que reintegrar la prima percibida hasta el momento, ni serían sancionadas. Cuando acabó el plazo correspondiente, el Ministerio de Industria apenas había recibido la renuncia de 982 instalaciones, que totalizaban una capacidad de 64,56 MW.

Mientras tanto, la campaña de inspección seguía su curso. A mediados del año 2011, había 1.282 plantas con el cobro de la prima suspendido (que representaban unos $228 \mathrm{MW}$ ). Pocos días después, saltó la noticia de que el Ministerio de Industria había admitido muchas de las alegaciones formuladas a la suspensión cautelar de la tarifa. Finalmente, la potencia considerada irregular se estimó en unos escasos 89 MW.

\section{El déficit tarifario y las normas aprobadas en 2009 y 2010}

A lo largo del año 2010, la discusión acerca del lastre económico de la tarifa fotovoltaica y, por extensión, el coste de promoción de las fuentes renovables, se mezcló con el asunto del llamado déficit tarifario. Dos hechos de naturaleza muy diferente 
que, por la fuerza de las circunstancias, quedaron de inmediato emparejados en el espinoso debate a propósito del costo del recibo de la luz y, de forma más general, las expectativas económico-financieras del sistema eléctrico español.

Cuando a finales de los años 90, el gobierno decidió controlar la evolución de las tarifas eléctricas con el objetivo de mantener comparativamente bajos los precios de la electricidad, la contrapartida fue el déficit tarifario, esto es, la diferencia entre las tarifas aprobadas por el gobierno y los costes (generación, distribución y otros) que la ley exige que sean sufragados con tales tarifas. Un déficit que toma la forma de una deuda de los consumidores con el sector eléctrico.

El monto del déficit tarifario ha sufrido importantes altibajos con el paso de los años. Nulo o de escasa magnitud a principios de la pasada década, a lo largo de su segunda mitad la recaudación por peajes fue quedando progresivamente rezagada con respecto a los costes que tenía que cubrir ${ }^{4}$. La impopularidad de un gran aumento de las tarifas eléctricas llevó a que se optase por desplazar hacia el futuro el pago del déficit tarifario, titulizándolo con aval del Estado desde 2010. Simultáneamente, se fueron tomando medidas para frenar su acumulación y avanzar en su reducción. Los recibos de la luz de los próximos años incorporarán, pues, el pago del servicio de la deuda eléctrica.

El debate acerca de qué medidas tomar ha sido encarnizado. Sin ánimo de entrar en detalles, el sector de las renovables ha exigido revisar ciertos capítulos de los gastos generales del sistema eléctrico, como las compensaciones por los sistemas extrapeninsulares o los costes de transición a la competencia; aplicar un impuesto sobre los windfall profits de las plantas nucleares e hidroeléctricas; o cargar fiscalmente a los hidrocarburos por las emisiones que conllevan. Los sectores aludidos, por su parte, han presionado en favor del recorte a la capacidad y a las tarifas y primas del régimen especial, especialmente las técnicas solares. En el momento de escribir estas páginas (principios de marzo de 2012), las principales medidas que se han tomado para frenar la acumulación del déficit han afectado a las técnicas renovables, tal como se explica en breve y en el próximo apartado.

Para empezar, el RD 1.565/2010 eliminaba, para las plantas fotovoltaicas inscritas según el RD 661/2007, la tarifa regulada más allá del vigésimo quinto año. Este punto fue rechazado de plano por las asociaciones patronales por considerarlo retroactivo. Además, esta norma incluía una rebaja de tarifas de carácter extraordinario, tal como mostraba el Gráfico 3. Este punto pone de manifiesto que el esquema retributivo del RD 1.578/2008, mucho más flexible que el anterior, estableció, sin embargo, una tasa de descenso de la tarifa que fue rebasada por el descenso del precio de los paneles en esos años. Una circunstancia que, por ejemplo, también ha afectado el régimen tarifario alemán.

${ }^{4}$ Capítulos como el transporte y la distribución eléctrica, el plan de residuos radioactivos, las primas del régimen especial, los planes de ahorro y eficiencia energética, los pagos por capacidad, las compensaciones extra-peninsulares, etc. y el servicio de la deuda tarifaria ya titulizada. A finales de 2011 el déficit tarifario acumulado se estimaba en 24 mil millones de euros. 
Simultáneamente fue tomando forma el RDL 14/2010 cuyo objetivo principal, aunque no el único, era incidir de lleno en las condiciones retributivas del RD 661/2007. Un asunto sin duda de mayor calado económico y legal. Así, pues, esta norma limitó las horas de funcionamiento de las instalaciones fotovoltaicas cuyos kWh generados se retribuirían a tarifa. El descenso fue más acusado para las plantas acogidas al RD 661/2007. También para estas instalaciones se extendió en tres años el plazo de veinticinco con retribución preferente que había establecido el RD 1565/2010, apenas un mes antes.

El sector insistió en que limitar el número de horas con derecho a tarifa era retroactivo. Sin embargo, varias sentencias del Tribunal Constitucional distinguen dos situaciones diferentes ${ }^{5}$ : por un lado, un cambio a posteriori de una norma vigente que obligase a la devolución de las cantidades ya percibidas, una medida que considera claramente retroactiva, y, por el otro, la modificación de las condiciones económicas sólo desde el momento que entre en vigor la nueva norma, sin que se reclamen las cantidades ya percibidas. En este segundo supuesto, la modificación tiene que respetar los plazos de adaptación y garantizar la viabilidad económica de las inversiones ya realizadas, según apunte la ley superior en que se apoya la regulación, en este caso, la Ley del Sector Eléctrico. Dos condicionantes que, dicho sea de paso, no están exentos de ambigüedad.

El sector se movilizó masivamente contra el RDL 14/2010. Especialmente activo fueron los fondos de inversión que, con el paso del tiempo, habían pasado a controlar parte del parque fotovoltaico. La prensa se hizo eco de presiones de varias embajadas a la CNE, al Ministerio de Industria y la Presidencia del Gobierno ${ }^{6}$. También el Comisario Europeo de la Energía afirmó que se podría abrir expediente a España, si la nueva regulación ponía en peligro los objetivos de generación renovable fijados por las directivas de la UE.

No existe o no se ha publicado un estudio riguroso sobre el impacto del RDL 14/2010 sobre la rentabilidad de las plantas fotovoltaicas. No obstante, en ASIF (2011, pp. 29-30) se expone, de forma un tanto confusa, que una planta con dos ejes de seguimiento, una relación potencia pico/potencia nominal de 1,2, con 2.300 horas de actividad y localizada en la zona climática IV (básicamente la mitad sur del país), sufre un descenso próximo al 20 por 100 de sus ingresos anuales. Por el contrario, si la relación de potencias es 1 , la merma de ingresos es del 5 por 100. Como término medio, esta fuente estima un descenso del 10 por 100. Si se trata de plantas acogidas al RD 661/2007, la disminución, ahora en términos de rentabilidad, sería de casi un 40 por 100 y de un 25 por 100 , respectivamente.

En otro orden de cosas, a mediados de 2010, de acuerdo con lo dispuesto en la Directiva 2009/28/CE de Energías Renovables, se redactó el Plan de Acción Nacio-

${ }^{5}$ Por ejemplo, las sentencias del Tribunal Constitucional 42/1986 del 10 de abril de 1986, publicada el 29 de abril, y 227/1988 del 29 de noviembre de 1988, publicada el 23 de diciembre.

${ }^{6}$ Directivos de varios fondos internacionales, con más de 3 mil millones de euros invertidos en plantas fotovoltaicas, se dirigieron por carta al Presidente del Gobierno instándole a que la retribución no fuese recortada (Cinco días, 21/6/2010, portada y p. 3). 
nal de Energías Renovables de España (PANER, 2010). En él se indicaba que, para 2020, las energías renovables deberían de aportar el 22,7 por 100 de la energía final bruta consumida. En el mix de generación eléctrica renovable, la fotovoltaica y la solar termoeléctrica aportaban el 3,6 y 3,8 por 100, respectivamente. A partir de 2015, el PANER preveía una penetración creciente de la energía fotovoltaica procedente de instalaciones para autoconsumo conectadas a la red. En el caso de la solar termoeléctrica, la capacidad acumulada en 2020 se estimaba en 5.079 MW.

El PANER, junto con el dictamen de la Subcomisión de Energía del Congreso, marcaron las líneas maestras del Plan de Energías Renovables (2011-2020) (PER, 2011). En éste la capacidad instalada fotovoltaica descendía hasta los 7.250 MW, esto es, 1.117 MW menos que los fijados en el PANER. También descendía la capacidad solar termoeléctrica: la meta para 2020 pasaba a ser 4.800 MW (PER, 2011, pp. 19-20). De todas formas, la potencia solar termoeléctrica subía 7,6 veces a lo largo de la década, mientras que la fotovoltaica no llegaba ni a duplicarse.

\section{La regulación de la generación solar termoeléctrica}

A lo largo de los años considerados, las vicisitudes experimentadas por el sector solar termoeléctrico tuvieron un cariz muy diferente a las del fotovoltaico. Aunque el combustible sea el mismo, se trata de técnicas sin relación entre sí. Además, el proceso de maduración de sus proyectos es mucho más largo y el perfil empresarial del sector muy distinto.

Las bases de la regulación de la generación solar termoeléctrica se establecieron en los RD 2818/1998 y 436/2004. Aunque nada decían acerca de las diversas tipos de plantas, sí abordaban la cuestión de la hibridación. Por su parte, el RD 661/2007 establecía la siguiente tarifa, sin distinción de técnicas o potencias: los primeros 25 años, 26,9375 cts $\square / \mathrm{kWh}$ y para el resto, $21,5498 \mathrm{cts} \square / \mathrm{kWh}$. Una vez autorizada, esta tarifa sería actualizada todos los años menos una reducción igual a la fijada para las tarifas fotovoltaicas. Sin embargo, se abrió la opción de que los productores solares termoeléctricos vendiesen la electricidad en el mercado mayorista, percibiendo el precio final horario medio de éste más una prima $(\lambda)$. La retribución total $\left(p_{T}\right) \mathrm{y}$ el valor de la prima serían calculados, hora tras hora, según el patrón que muestra el Gráfico 5. 


\section{GRÁFICO 5}

\section{LA PRIMA VARIABLE DE LA GENERACIÓN SOLAR TERMOELÉCTRICA}

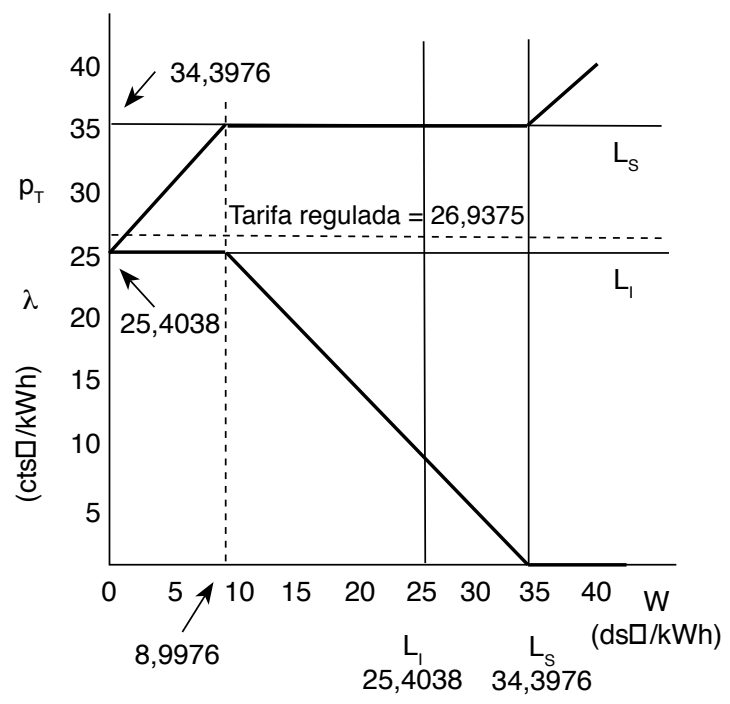

El productor percibe, como mínimo, 25,4 cts $\square / \mathrm{kWh}$ (o límite inferior, $L_{I}$, que, en el caso solar termoeléctrico coincide con la prima de referencia). Si el precio de mercado supera los $8,9976 \mathrm{cts} \square / \mathrm{kWh}$, la suma de éste más la prima de referencia rebasa el límite superior $\left(L_{S}\right)$. Por ello, la prima a percibir va descendiendo paulatinamente. El resultado es que el kWh se retribuye al límite superior para un amplio recorrido de valores del precio de mercado. Un límite superior que está por encima de la tarifa. No resulta, pues, sorprendente que las plantas solares termoeléctricas hayan optado por la prima variable. Finalmente, si el precio de mercado supera el límite superior, los productores no perciben prima. El precio cobrado es el del mercado mayorista.

Como ya sucedió con la técnica fotovoltaica, a pesar del apoyo ofrecido por el RD 661/2007, durante meses nada significativo ocurrió. Los proyectos con vocación comercial llegaban con cuentagotas. No obstante, a lo largo de 2008 el sector empezó a ser noticia. Una tendencia que se reforzó en 2009, cuando pasaron a ser comunes las declaraciones de diversas empresas, muchas de ellas grandes firmas de la construcción y de la ingeniería, que decían estar desarrollando proyectos de plantas comerciales (San Miguel, Del Río y Hernández, 2010). Esta aceleración fue incentivada, por una parte, por la existencia del objetivo de $500 \mathrm{MW}$ que preveía el RD 661/2007. Una vez alcanzado el 85 por 100 de esta cifra, empezaba un plazo transitorio cuya conclusión sería, probablemente, una rebaja de las primas. Por la otra, el RDL 6/2009 extendió el requisito de inscripción en el Registro de preasignación a todas las técnicas del régimen especial.

A finales de 2009, se contabilizaban proyectos en curso de redacción o tramitación que totalizaban unos 4.000 MW, aunque sólo la mitad había depositado el aval para optar a la pre-asignación. Un momento clave fue el 13 de noviembre de 2009 , 
cuando el Consejo de Ministros aprobó una previsión de 2.400 MW solares termoeléctricos adicionales, hasta finalizar 2013. Ello suponía unas 55 nuevas plantas. No se abordó, sin embargo, el tema de la retribución, que continuaría ajustada al RD 661/2007, aunque con algún tipo de recorte. Las negociaciones entre el sector y el Ministerio de Industria dieron paso al RD 1.614/2010. Como punto principal, esta norma limitaba el número de horas de funcionamiento con derecho a prima, diferenciándose hasta ocho modalidades de generación solar termoeléctrica. Estos límites no serían revisados para toda su vida útil en el caso de las plantas inscritas con carácter definitivo con anterioridad a la entrada en vigor del RDL 6/2009, esto es, el 7 de mayo de 2009, así como algunas otras no definitivamente inscritas en la fecha recién indicada pero que cumplían con ciertos requisitos documentales y que habían depositado el aval correspondiente. Además, el artículo 4 suprimía la correspondiente revisión de tarifas dispuesta en el artículo 44.3 del RD 661/2007. Así pues, la negociación entre las autoridades y el sector consistió en garantizar la inscripción para los proyectos más maduros todavía pendientes, junto con una atractiva retribución para toda su vida útil, a cambio de fijar un límite al número de horas con derecho a prima y una reducción en el número de MW solares termoeléctricos que, según se había pregonado, estaban desarrollándose.

Para terminar con este apartado, el Gráfico 6 muestra la expansión mensual de la capacidad instalada solar termoeléctrica desde 2007 a 2011, mientras que el Cuadro 4 reúne los datos básicos relativos a la generación solar termoeléctrica (en ambos casos, elaboración propia en base a datos de la $\mathrm{CNE}$ ).

\section{GRÁFICO 6}

CAPACIDAD SOLAR TERMOELÉCTRICA (2007-2011)

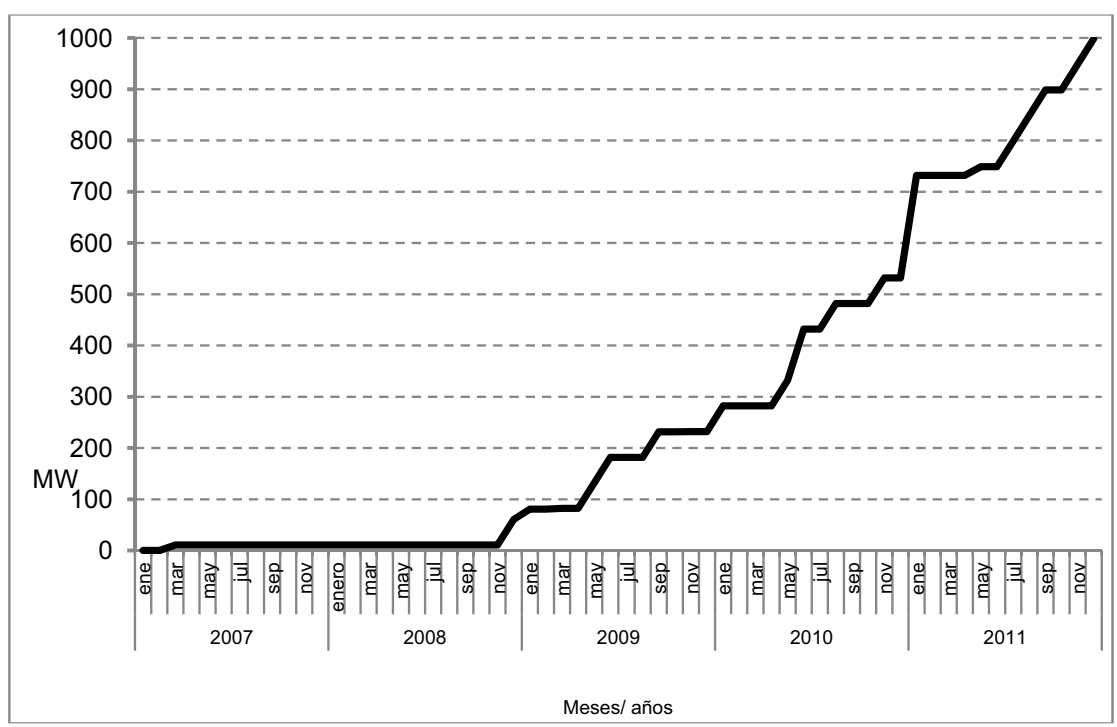




\section{CUADRO 4}

DATOS BÁSICOS DE LA GENERACIÓN SOLAR TERMOELÉCTRICA

\begin{tabular}{|l|c|c|c|c|}
\hline & $\begin{array}{c}\text { Potencia operativa } \\
\text { (MW) }\end{array}$ & $\begin{array}{c}\text { Energía vendida } \\
\text { (GWh) }\end{array}$ & $\begin{array}{c}\text { Prima equivalente } \\
\text { (miles } \boldsymbol{\epsilon} \text { ) }\end{array}$ & $\begin{array}{c}\text { Prima } \\
\mathbf{\epsilon} / \mathbf{M W h}\end{array}$ \\
\hline 2009 & 232 & 17 & $4.496,5$ & 264,5 \\
\hline 2010 & 532 & 692 & $184.872,0$ & 267,1 \\
\hline 2011 & 999 & 1.777 & $426.274,0$ & 239,9 \\
\hline $2012(\mathrm{p})$ & $\sim 1.500$ & $<4.800$ & $>553.000,0$ & - \\
\hline
\end{tabular}

Para los años considerados en el cuadro y su comparación con los datos del Gráfico 2, en términos de prima equivalente el coste del $\mathrm{kWh}$ solar termoeléctrico es, aproximadamente, 0,6 veces el fotovoltaico.

\section{La moratoria de enero de 2012 y el informe del 7 de marzo de la CNE}

El pasado 27 de enero de 2012 el Consejo de Ministros aprobó, por procedimiento de urgencia, el RDL 1/2012, que, amparándose en la necesidad imperiosa de frenar la acumulación de más déficit tarifario, suspende, por un periodo no definido, ${ }^{7}$ la retribución preferente a toda nueva instalación solar y eólica. En concreto, se suprimen tarifas, primas y los complementos por eficiencia y por energía reactiva. Una supresión que afecta a cualquier instalación que, para el día 28 de enero, fecha de entrada en vigor del RDL, todavía no estuviese inscrita en el registro de preasignación. También todos aquellos promotores que, de forma voluntaria, opten por no ejecutar su proyecto aunque lo tengan ya inscrito, podrán recuperar los avales depositados.

El artículo 4 suspende el procedimiento de preasignación para todas las técnicas del régimen especial. Además, el artículo 4.2 se refiere de forma expresa a la interrupción de las convocatorias del cupo fotovoltaico para 2012 y años sucesivos.

En la exposición de motivos, párrafos 7 a 9, se declara que las medidas del RDL no ponen en riesgo el incumplimiento por parte de España de los compromisos asumidos en el marco del triple 20 fijado por la UE. Por su parte, el párrafo 12 hace referencia a ciertas reformas regulatorias en estudio favorables al net-metering, aunque no se concreta nada ni se fijan plazos. También merecen ser destacados los párrafos 13 y 14 que hablan de la necesidad de diseñar un nuevo modelo retributivo. Su lectura parece sugerir que, cuando se reactive la política de fomento de las renovables, la asignación de potencia se hará mediante subastas. Más concreta es la norma cuando detalla que son $550 \mathrm{MW}$ la potencia fotovoltaica afectada, esto es, el cupo de 2012, a la vez que permite la puesta en marcha de las plantas solares termoeléctricas ya registradas (unos 1.600 MW). Una disposición que no recoge la petición de UNESA de establecer una moratoria total para este tipo de generación.

\footnotetext{
${ }^{7}$ Aunque se afirma que la supresión es de carácter temporal, dado que queda supeditada a la resolución del déficit tarifario, puede suponerse que la suspensión se extenderá, cuanto menos, un par de años.
} 
La impresión general es que, para el legislador, las técnicas solares (y la eólica) son las principales responsables de la dinámica del déficit tarifario. Aunque el mayor peso se reserve para la fotovoltaica, el RDL 1/2012 perjudica comparativamente más a la solar termoeléctrica, dado que se reduce a la mitad el techo de potencia pactado en 2010. Por lo que se refiere a la técnica fotovoltaica, a la drástica reducción de la actividad sufrida en 2009, se añade ahora su parálisis total sine die. Si bien en términos comparativos el daño adicional es limitado, en valores absolutos el sector cae en un estado vegetativo.

Sin embargo, no está claro que, con las medidas contenidas en esta norma, se resuelva el problema del crecimiento sostenido del déficit tarifario. Por una parte, la retribución a las plantas ya operativas se actualiza todos los años y, por la otra, el déficit está provocado por causas muy diversas, algunas muy alejadas de la actividad de las renovables. Por lo tanto, habrá que esperar a saber qué otras medidas se toman para frenar su acumulación y, a continuación, reducirlo.

Antes de terminar con este apartado, hay que referirse al informe recién publicado por la Comisión Nacional de la Energía (CNE), relativo a las medidas para garantizar la sostenibilidad económico-financiera del sistema eléctrico español o, dicho de forma más directa, para conseguir frenar la acumulación del dèficit tarifario (CNE, 2012). Entre las muchas iniciativas que se proponen, hay un grupo que afecta a la generación solar termoeléctrica, especialmente a los aproximadamente $1.600 \mathrm{MW}$ ya preasignados pero sin autorización definitiva. Las medidas, sean a corto o medio plazo, más destacadas son las siguientes:

1. Rebajar el porcentaje de hibridación al 5 por 100. Con ello, los MWh generados con el recurso a los combustibles fósiles por encima de este nivel, pasan a ser vendidos a precios del mercado mayorista (ibídem, pp. 23 y 24).

2. Rebajar un 12 por 100 las primas ya que el supuesto utilizado años atrás para el cálculo de la rentabilidad, consideró precios del pool superiores a los vigentes (ibídem, p. 23).

3. Para los inmediatos próximos años, retribuir la energía generada por debajo de la prima vigente. Entrados en la segunda mitad de la presente década, las primas se cobrarían con un plus. A cambio se otorgaría a los titulares de las plantas una compensación por la inversión realizada cuyas anualidades, valoradas al 8 por 100, se extenderían un mínimo de 15 años (ibídem, pp. 52-53 y 115-119).

4. Desarrollar una normativa en la que el límite superior (cap) de la prima sea el precio más alto que pueda percibir el generador. En la legislación vigente, si el precio del pool supera este límite, la planta percibe el precio del mercado. En la modificación propuesta, los precios mayoristas por encima del límite superior darían paso a una prima negativa. Los generadores deberían devolver lo percibido por encima del cap (ibídem, pp. 82-83). 
A ello habría que añadir la propuesta de aumentar, con carácter general, las deducciones que se aplican a la actualización anual de las tarifas (ibídem, pp. 22-23)

\section{Conclusiones}

La líneas maestras de la regulación solar apenas variaron entre la última década del siglo pasado y finales de septiembre de 2008. El aspecto más significativo fue que los sucesivos reales decretos fueron, progresivamente, otorgando mayor seguridad a la inversión en fuentes renovables.

En el RD 661/2007 el legislador, consciente del comparativamente mayor coste de la generación fotovoltaica, fijó una potencia objetivo muy cercana a la ya instalada. Con ello se confiaba en mantener bajo control la expansión de su coste tarifario. Sin embargo, esta norma establecía paradójicamente un periodo transitorio muy largo para una técnica modular y relativamente fácil de instalar. Un lapso que, además, terminaba de forma abrupta: las tarifas preferentes desaparecían. Parece que nadie se percató de las potenciales consecuencias negativas de este fallo regulatorio. Se hicieron evidentes cuando la inversión en plantas solares (y eólicas) se convirtió en un producto financiero muy atractivo (por su rentabilidad y seguridad) en la etapa final de un ciclo expansivo, casi sin precedentes en la economía española. Fue esta circunstancia la que destapó los efectos nada inocuos del fallo regulatorio indicado. Este defecto de diseño se convirtió, pues, en el talón de Aquiles de una norma que, justo es reconocerlo, merece ser destacada por su exhaustividad y rigor.

Aunque sea absurdo exigir que un esquema regulatorio sea capaz de prever todos los escenarios, todas las contingencias posibles, sí que debería de estar capacitado para adaptarse, sin dilación y con encaje preciso, a los cambios propios del objeto regulado (por ejemplo, un descenso de los costes por un avance técnico inesperado) y/o factores exógenos al sector (como una modificación de la coyuntura que despierta un insospechado interés por la actividad en cuestión).

Una vez que las previsiones de capacidad instalada, y el concomitante coste tarifario, quedaron desbordados, los remedios estaban claros: establecer cupos para el control estricto del aumento de capacidad y proceder a instaurar mecanismos para sucesivas rebajas de las tarifas, tanto para las plantas ya operativas como las futuras. Estas medidas, sin embargo, acarreaban sus problemas. Por una parte, ponían en entredicho el cumplimiento de los objetivos del triple 20 establecidos por la UE y, por la otra, dificultaban enormemente la posibilidad de desarrollar un sector industrial fotovoltaico y solar termoeléctrico propio, aunque las principales empresas de ambos sectores iniciaron procesos de expansión internacional. De todas formas, el auténtico quebradero de cabeza era el monto de la retribución de las planta acogidas al RD 661/2007. Un grave problema económico y legal. Económico pues el recorte de las tarifas ponía en riesgo la viabilidad económica de las plantas y, por extensión, la recuperación de los fondos prestados. Legal por la posible retroactividad de esta 
medidas. Aunque no esté claro que así sea, es probable que ello derive en un largo y complejo pleito ante los tribunales.

Con el cambio de gobierno, la primera decisión ha sido la moratoria de toda nueva actividad de las principales técnicas renovables. Con ello España desaparece, en el corto plazo, del mercado fotovoltaico mundial (sólo un puñado de empresas tiene proyección internacional) mientras que las grandes firmas implicadas en la generación solar termoeléctrica se ven abocadas a confiar en la expansión exterior. A medio plazo, la suerte del sector solar, tanto en España como en el resto del mundo, queda sujeta al hecho de que el descenso de los costes, tanto de producción como de almacenamiento (de electricidad, de calor), sea de tal magnitud que inversionistas y particulares, sin apenas ayudas económicas de ningún tipo, encuentren atractivo instalar placas fotovoltaicas en el suelo o en los tejados, o crezca de forma imparable el número de plantas solares termoeléctricas. No hay duda de que, con ello, la generación solar ganará espacio tanto a costa de la generación eléctrica tradicional, como del resto de la renovable. Si la competitividad del $\mathrm{kWh}$ solar se alcanza por el aumento de los costes de generación convencional, debido al incremento del precio de los hidrocarburos y/o el establecimiento de tasas por emisiones, las expectativas del sector solar mejorarán sólo de forma limitada, habida cuenta de que otras fuentes renovables seguirán en cabeza.

\section{Referencias bibliográficas}

[1]ASIF (2011): Hacia el crecimiento sostenido de la fotovoltaica en España. Informe anual 2011, Madrid, Asociación de la Industria Fotovoltaica. Documento disponible en www.asif.org.

[2] CNE (2008): Informe 30/2008 de la CNE en relación a la propuesta de real decreto de retribución de la actividad de producción de energía eléctrica mediante tecnología solar fotovoltaica para instalaciones posteriores a la fecha límite de mantenimiento de la retribución del Real Decreto 661/2007, de 25 de mayo, para dicha tecnología, Comisión Nacional de Energía, Madrid, 29 de julio de 2008. Documento disponible en www.cne.es.

[3] CNE (2012): Informe sobre el sector energético español, Comisión Nacional de Energía, Madrid, 7 de marzo de 2012. Documento disponible en www.cne.es.

[4] DEL RÍO, P. Y GUAL, M. A. (2007): «An integrated assessment of the feed-in tariff system in Spain», Energy Policy, 35, pp. 994-1012.

[5] DEL RÍO, P. (2008): «Ten years of renewable electricity policies in Spain: An analysis of successive feed-in tariff reforms», Energy Policy, 36, pp. 2917-2929.

[6] DE LA HOZ, J. et al. (2010): «Promotion of grid-connected photovoltaic systems in Spain: Performance analysis of the period 1998-2008", Renewable and Sustainable Energy Reviews, 14, pp. 2547-2563.

[7] FUNK, J. et al. (2010): «Toward a Sunny Future? Global Integration in the Solar PV Industry», Working Paper Series, pp. 10-6, World Resources Institute/Peterson Institute for International Economics, Washington DC. Documento disponible en www.wri.org.

[8] J ÄGER-WALDAU, A. (2007): PV Status Report 2007. Research, Solar Cell Production and Market Implementation of Photovoltaics (JRC Technical Notes, EUR 23018 EN), 
Institute for the Environment and Sustainability/Joint Research Centre/European Communities, Office for Official Publications of the European Communities, Luxembourg. Documento disponible en http://ies.jrc.ec.europa.eu.

[9] L 54/1997: Ley 54/1997, de 27 de noviembre, del Sector Eléctrico, Boletín Oficial del Estado, núm. 285, de 27 de noviembre de 1997.

[10] MIR, P. (2009): Economía de la generación fotovoltaica, Lleida, Editorial Milenio.

[11] PANER (2010): Plan de Acción Nacional de Energías Renovables en España, Madrid, IDEA/Ministerio de Industria. Documento disponible en www.idae.es.

[12] PER (2011): Plan de Energías Renovables (2010-2020), Madrid, IDEA/Ministerio de Industria. Documento disponible en www.idae.es.

[13] RD 436/2004: Real Decreto 436/2004, de 12 de marzo, por el que se establece la metodología para la actualización y sistematización del régimen jurídico y económico de la actividad de producción de energía eléctrica en régimen especial, Boletín Oficial del Estado, núm. 75, de 27 de marzo de 2004, pp. 13217-13238.

[14] RD 661/2007: Real Decreto 661/2007, de 25 de mayo, sobre la regulación de la actividad de producció de energía eléctrica en régimen especial, Boletín Oficial del Estado, núm. 126, de 26 de mayo de 2007, pp. 22846-22886.

[15] RD 1003/2010: Real Decreto 1003/2010, de 5 de agosto, por el que se regula la liquidación de prima equivalente a las instalaciones de producción de energía eléctrica de tecnología fotovoltaica en régimen especial, Boletín Oficial del Estado, núm. 190, de 6 de agosto de 2010, pp. 68610-68615.

[16] RD 1565/2010: Real Decreto 1565/2010, de 19 de noviembre, por el que se regulan y modifican determinados aspectos relativos a la actividad de producción de energía eléctrica en régimen especial, Boletín Oficial del Estado, núm. 283, de 23 de noviembre de 2010, pp. 97428-97446.

[17] RD 1578/2008: Real Decreto 1578/2008, de 26 de septiembre, sobre la retribución de la actividad de producción de energía eléctrica mediante tecnología solar fotovoltaica para instalaciones posteriores a la fecha de vigencia de la retribución del Real Decreto 661/2007, de 25 de mayo, Boletín Oficial del Estado, núm. 234, de 27 de septiembre de 2008, pp. 39117-39125.

[18] RD 1614/2010: Real Decreto 1614/2010, de 7 de diciembre, por el que se regulan y modifican determinados aspectos relativos a la actividad de producción de energía eléctrica a partir de tecnologías solar termoeléctrica y eólica, Boletín Oficial del Estado, núm. 298, de 8 de diciembre de 2010, pp. 101853-101859.

[19] RD 2818/1998: Real Decreto 436/2004, de 23 de diciembre, sobre producción de energía eléctrica por instalaciones abastecidas por recursos o fuentes de energía renovables, residuos y cogeneración, Boletín Oficial del Estado, núm. 312, de 30 de diciembre de 1998, pp. 44077-44089.

[20] RDL 6/2009: Real Decreto-ley 6/2009, de 30 de abril, por el que se adoptan determinadas medidas en el sector energético y se aprueba el bono social, Boletín Oficial del Estado, núm. 111, de 7 de mayo de 2009, pp. 39404-39419.

[21] RDL 14/2010: Real Decreto-ley 14/2010, de 23 de diciembre, por el que se establecen medidas urgentes para la corrección del déficit tarifario del sector eléctrico, Boletín Oficial del Estado, núm. 312, de 24 de diciembre de 2010, pp. 106386-106394.

[22] RDL 1/2012: Real Decreto-ley 1/2012, de 27 de enero, por el que se procede a la supresión de los procedimientos de preasignación de retribución y a la supresión de los 
incentivos económicos para nuevas instalaciones de producción de energía eléctrica a partir de cogeneración, fuentes de energía renovables y residuos, núm. 24 , de 28 de enero de 2012, pp. 8068-8072.

[23] SAN MIGUEL, G.; DEL RÍO, P. Y HERNÁNDEZ, F. (2010): «An update of Spanish renewable energy policy and achievements in a low carbon context», Journal of Renewable and Sustainable Energy, 2 (3), pp. 031007-1/17. 


\section{SUSCRIPCIÓN ANUAL}

\begin{tabular}{|c|c|c|c|}
\hline \multicolumn{4}{|c|}{ CUADERNOS ECONÓMICOS DE INFORMACIÓN COMERCIAL (2 NÚMEROS) } \\
\hline & $\begin{array}{c}\text { ESPAÑA } \\
\mathbf{1} \text { año }\end{array}$ & $\begin{array}{c}\text { UNIÓN EUROPEA } \\
\mathbf{1} \text { año }\end{array}$ & $\begin{array}{c}\text { RESTO DEL } \\
\text { MUNDO } \\
\mathbf{1} \text { año }\end{array}$ \\
\hline SUSCRIPCIÓN & $30,00 €$ & $40,00 €$ & $40,00 €$ \\
\hline $\begin{array}{c}\text { Gastos de envío } \\
\text { España }\end{array}$ & $2,36 €$ & $10,04 €$ & $16,10 €$ \\
\hline $\begin{array}{c}\text { Más 4\% de IVA. } \\
\text { Excepto Canarias, Ceuta y Melilla }\end{array}$ & $1,29 €$ & & \\
\hline TOTAL & $\mathbf{3 3 , 6 5} €$ & $\mathbf{5 0 , 0 4} €$ & $\mathbf{5 6 , 1 0} €$ \\
\hline & $\square$ & $\square$ & $\square$ \\
\hline
\end{tabular}

\section{EJEMPLARES SUELTOS}

\begin{tabular}{|c|c|c|c|}
\hline \multicolumn{4}{|c|}{ CUADERNOS ECONÓMICOS DE INFORMACIÓN COMERCIAL } \\
\hline & $\begin{array}{c}\text { ESPAÑA } \\
1 \text { ejemplar }\end{array}$ & $\begin{array}{c}\text { UNIÓN EUROPEA } \\
1 \text { ejemplar }\end{array}$ & $\begin{array}{c}\text { RESTO DEL } \\
\text { MUNDO } \\
\mathbf{1} \text { ejemplar }\end{array}$ \\
\hline NÚMERO SUELTO & $18,00 €$ & $23,00 €$ & $23,00 €$ \\
\hline $\begin{array}{c}\text { Gastos de envio } \\
\text { España }\end{array}$ & $1,18 €$ & $5,02 €$ & $8,05 €$ \\
\hline $\begin{array}{c}\text { Más 4\% de IVA. } \\
\text { Excepto Canarias, Ceuta y Melilla }\end{array}$ & $0,77 €$ & & \\
\hline TOTAL & $\mathbf{1 9 , 9 5} €$ & $\mathbf{2 8 , 0 2} €$ & $\mathbf{3 1 , 0 5} €$ \\
\hline
\end{tabular}

\section{DATOS}

Nombre y apellidos

Empresa

Domicilio

D.P.

N.I.F.

Teléf
Población

Fax $\square$ Transferencia a la cuenta del Centro de Publicaciones del Ministerio de Industria, Turismo y Comercio. BBVA. $P^{0}$ de la Castellana, 148. 28046 MADRID (ESPAÑA) CÓDIGO CUENTA CLIENTE: 0182-9091-52-0200000597
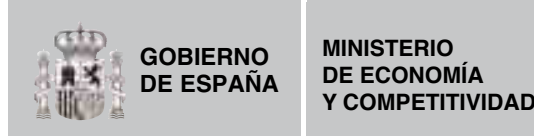

SUBSECRETARÍA

SECRETARÍA GENERAL TÉCNICA

SUBDIRECCIÓN GENERAL

DE DESARROLLO NORMATIVO,

INFORMESY PUBLICACIONES

CENTRO DE PUBLICACIONES

Información y venta directa:

Calle Panamá, 1. Vestíbulo. 28071 Madrid. Teléfono 913497605 / 3494968

Suscripciones y ventas por correspondencia:

Calle Panamá, 1. Planta 0. 28071 Madrid. Teléfono 9134951 29. Fax: 913494485

Suscripciones a través de la página web del Ministerio de Industria, Turismo y Comercio:

http://www.revistasice.com/RevistasICE/Suscripciones/pagFormulario.htm 